\title{
Design \& investigation of 10x10 gbit/s MDM over hybrid FSO link under different weather conditions and fiber to the home
}

\author{
Alaan Ghazi ${ }^{1}$, S. A. Aljunid ${ }^{2}$, Awab Noori ${ }^{3}$, Syed Zulkarnain Syed Idrus ${ }^{4}$, C. B. M. Rashidi ${ }^{5}$, Aras Al-Dawoodi ${ }^{6}$ \\ ${ }^{1,4}$ School of Human Development \& Techno Communication, University Malaysia Perlis, Malaysia \\ ${ }^{1,2,5}$ School of Computer and Communication Engineering, University Malaysia Perlis, Malaysia \\ ${ }^{3}$ Optical Computing, Technology Laboratory, School of Computing, University Utara Malaysia, Malaysia \\ ${ }^{6}$ Computer Science Department, College of Computer Science and Information Technology, Kirkuk University, Iraq
}

\begin{tabular}{l} 
Article Info \\
\hline Article history: \\
Received Oct 18, 2018 \\
Revised Nov 25, 2018 \\
Accepted Dec 12, 2018 \\
\hline
\end{tabular}

\section{Keywords:}

Bit-error rates

Eye diagram

Fiber to the home

Free space optics

Mode division multiplexer

\begin{abstract}
In this paper, we design and investigate 10-channels of mode division multiplexer (MDM) over hybrid free-space optics (FSO) link in several weather conditions to achieve the maximum possible medium range and fiber to the home (FTTH) for high bandwidth access networks. System capacity can be effectively increased with the use of MDM over hybrid FSO-FTTH. In this study, a 10-channel MDM over FSO-FTTH system has been analyzed in different weather conditions that operate at $1550 \mathrm{~nm}$ wavelength. The simulated system has transmitted $100 \mathrm{Gbit} / \mathrm{s}$ up for a distance of 3200 meters FSO in superbly clear weather condition. It also transmitted $100 \mathrm{Gbit} / \mathrm{s}$ up for a distance of 650 meters FSO during heavy rain. The validation of this study is measures based on eye diagrams bit-error rates (BER) that have been analyzed.
\end{abstract}

Copyright $\odot 2019$ Institute of Advanced Engineering and Science. All rights reserved.

\section{Corresponding Author:}

Alaan Ghazi,

School of Human Development \& Techno Communication,

University Malaysia Perlis, Malaysia,

Kampung Kubang, 02600 Arau, Perlis

Email: alaanghazi@gmail.com

\section{INTRODUCTION}

The proliferation of free-space optics (FSO) has seen the network technology and its prospects soar as the next-generation wireless broadband [2]. The wireless telecommunication system of FSO utilizes a transmission medium of free space to transmit optical data at high bit rates. In comparison with the conventional wireless technologies, this optical wireless connection possesses several positive benefits which includes high bandwidths, cost-efficiency, long transmission distance, interference immunity, license-free operation, amongst others. From its basic development, the technology has been improved remarkably in recent years. FSO uses a similar optical transceiver, like that of optical communication technology, and it operates through free space, in full-duplex mode.

More importantly, its installation can be very swift at a very low cost. However, it is exposed to various effects of noise in different weather conditions [1]. Concurrently, the amount of data that is transmitted keeps increasing. It could be possibly handled by considering several major at the transmitter like minding the modulation techniques [2], suitable light source, estimation of transmitting power levels, and wavelength transmission [3]. Accordingly, the demand for bandwidth in support of numerous applications keeps increasing too, over the years [4]. When the bandwidth is expanded, the transmission capacity of the FSO is also accordingly enhanced, which is necessary in the current video-based information dissemination world. In addition, hybrid FSO-fiber promises to provide unlimited integrated services at a substantially high bandwidth and also to support reliability in smart cities $[5,6]$. 
Nowadays, private enterprises and government have begun to recognize the significance and potentials of fiber-to-the-home (FTTH) and consequently, countless homes and businesses have exponentially connected over optical fiber [5, 7, 8]. In order to facilitate medium-sharing infrastructure within FTTH wireless networks, quite a number of multiple-input multiple-output (MIMO) systems have been suggested as options for FTTH access networks for the smart city.

The exponential increase of data traffic in FTTH has inspired researchers across the world to explore various multiplexing techniques within access networks to increase its capacity and transmission distance. Most of the multiplexing schemes for access networks are based on time division multiplexer (TDM) access [9], wavelength division multiplexer (WDM) access [10] have been used besides frequency division multiplexer (OFDM) access [11] and optical code division multiple (OCDM) access [5]. Mode division multiplexer (MDM) is a new scheme that has attracted remarkable attention as an innovative technology which increases the data rate $[6,7,12]$.

Moreover, amongst many of the multiplexing schemes, MDM is a swiftly emerging as a potential candidate for increasing the aggregate bandwidth of current optical fiber systems. MDM multiplexing supporting several modes propagate through optical fibers transmission. An MDM is an optical communication method where spatial modes are utilized as information channels carrying independent data streams. In this paper, we designed 10 channels of MDM over hybrid FSO and FTTH link under different weather conditions to achieve the maximum possible medium range for high bandwidth wireless access networks for smart cities as shown in Figure 1.

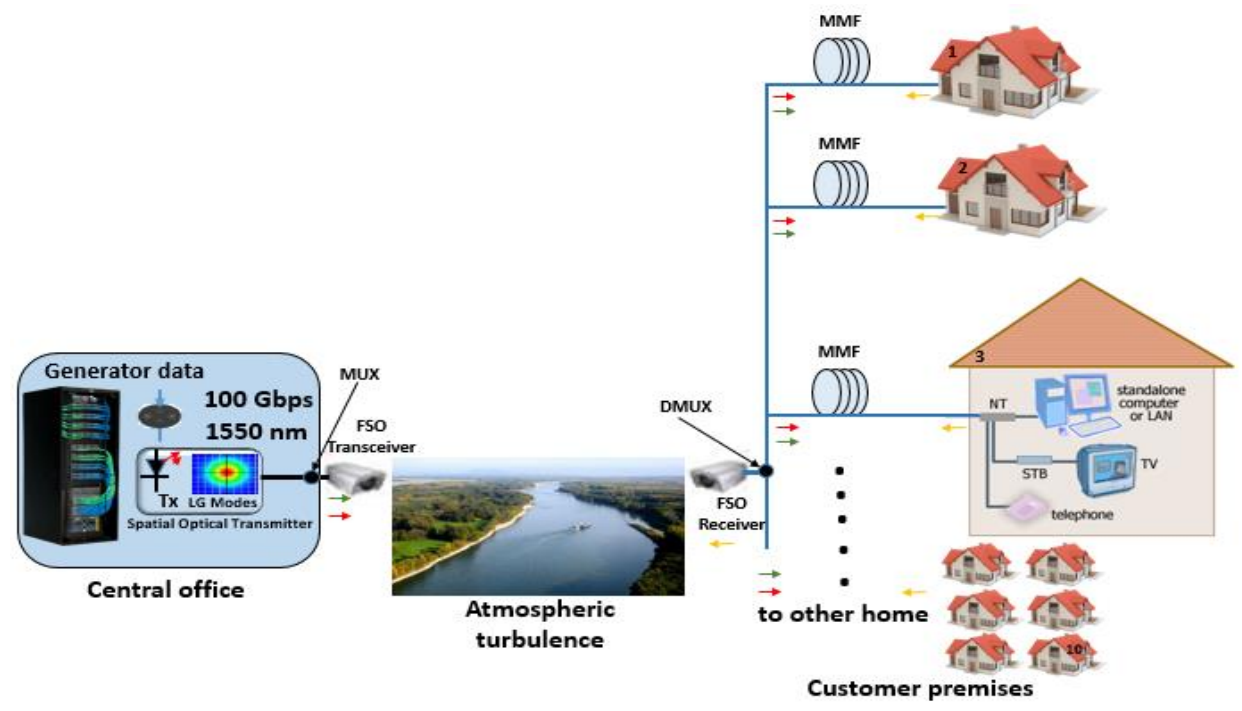

Figure 1. Architecture of hybrid FSO link and fiber to the home

This study tests the technology in various weather conditions to determine the effects on its performance. In MDM, different modes are used to facilitate the propagation of a various number of modes on different channels generated by various techniques based on optical signal processing [13, 14], few-mode fiber [11, 15] and modal decomposition methods [16, 17] MDM based on switching [18], also multiplexer [12] over optical communication. Moreover, electronic and photonic equalization schemes [19-22] could possibly be explored and integrated into the system. The other scheme of optimization soft computing algorithm [23-25] that can be used in optical communication to improve nonlinearly distortion. Despite the benefits of FSO technology, it is also challenged by several weaknesses to its bandwidth.

Evidently, alignment requirement that are strict as well as hostile or harsh atmospheric weather conditions are amongst the main challenges [1]. Basically, FSO's performance could be reinforced and buttressed by adopting modulation techniques [2] and also applying estimation of transmitting power levels as well as wavelengths [3]. In this study, the MDM of LG modes is designed with varying the diameters based on vertical-cavity surface emitting-laser (VCSEL). In the subsequent sections of this article, next section discusses the MDM simulation for LG modes over FSO; the following section outlines the outcomes of the MDM simulation and ends with a section that concludes the entire paper. 


\section{SYSTEM DESCRIPTION}

Hybrid free-space optics linked beneath various weather case to achieve the maximum possible medium range and Fiber-To-The-Home (FTTH) based on MDM system of 10-channels has been simulated and designed using Opt-system [26] software in as illustrated in Figure 1. The FSO -FTTH based on MDM system consists of four parts; Central office which is a transmitter, FSO channel, MMF channels, and receiver which is the customer premises. In the transmitter side, the special optical transmitter is used to generate data at 10-Gbit/s, and the electrical modulated is based on non-return to zero (NRZ) sequence, special optical transmitters that subcarrier over one wavelength $(1550 \mathrm{~nm})$, and it sends ten channels goes to ten Laguerre-Gaussian (LG) modes such as LG (0 0, 0 1, 0 2, $10,11,12,13,20,2$ 2, and 2 1) modes transmitted $100 \mathrm{Gbit} / \mathrm{s}$ long FSO under different weather conditions.

Furthermore, each special optical transmitter carries an array which emits one LG mode. Given 10 Gbit/s is transmitted based on one wavelength. Thus, with 10-channel based, the total data rate is $10 \times 10$ Gbit/s=100 Gbit/s over FSO channel is $3.2 \mathrm{~km}$ long under very clear weather condition and MMF. The parameters of receiver and transmitter apertures are set to $30 \mathrm{~cm}$, whilst the laser power set to $1 \mathrm{dBm}$, and beam divergence is $1 \mu \mathrm{rad}$ [12] based on various weather case. After FSO, the de-multiplexer is made to retrieve the signals from the FSO to ten different MMF.

Moreover, the MDM signals are then propagated through a 30m over MMF. The presumed value for peak-index 1.5 none, attenuation is $0.3 \mathrm{~dB} / \mathrm{km}$, operational-mode "parabolic" with consideration of modal coupling and after the MMF the ten special optical receivers were used to retrieve the signals. The measures and the performance based on bit-error rates (BER) and eye diagrams are discussed in the following section.

\section{RESULTS AND ANALYSIS}

The BER and eye diagram revealed significant results for the improvement of the bandwidth in the FSO system, as shown in Figure 2 and Figure 3 presented by the eye diagram where the eye openings for all channels on the ten LG modes have been widened for very clear weather condition. Meanwhile, the performance matrix based on BER was measured in different weather conditions as shown in Figure 4 . This paper presents results of performance of simulated 10-channel MDM of LG mode FSO in different weather conditions. The system runs at the maximum link range with high-speed data rate up to $100 \mathrm{Gbit} / \mathrm{s}$. The performance of the system is measured for heavy haze, light haze, heavy rain, light rain, clear sky, and very clear conditions with good BER. Detailed results are shown in Table 1. Weather condition affects the performance significantly.

Particularly, it was found that the maximum link can be carried out up to 650 meters only in heavy rain condition. In contrast, it could go up to 3200 meters in clear weather condition. This study discovers significant results by improving the BER and eye diagram based on MDM. Obviously, the performance improves along with the improvement of the bandwidth of optical wireless communication system. Furthermore, Figure 2 and Figure 3 represent the eye diagram with the ten LG modes in very clear weather condition. Obviously, the eye diagrams exhibit good results.

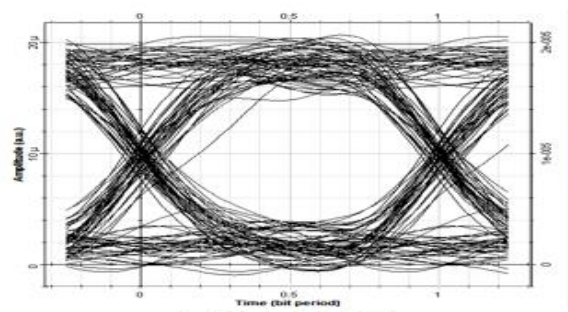

(a) Mode (LG O O)

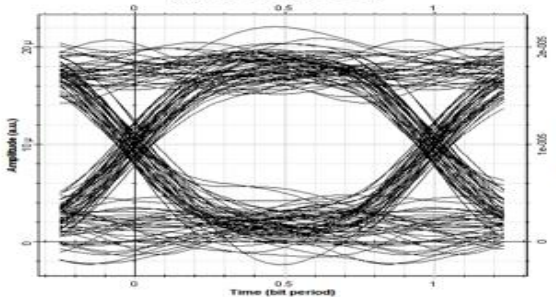

(c) Mode (LG 0 2)

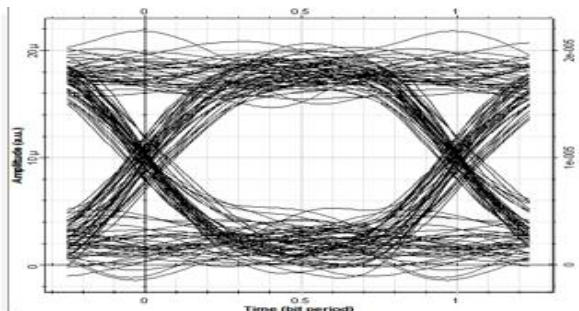

(b) Mode (LG 0 1)

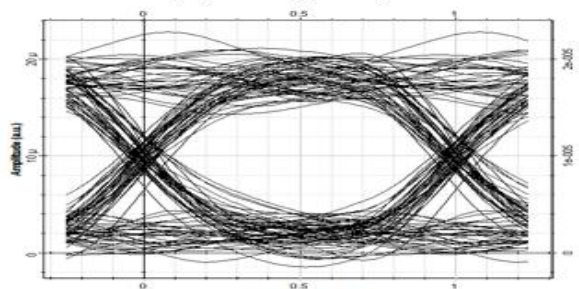

(d) Mode (LG 10 )

Figure 2. Representing the eye diagrams of first four channels 


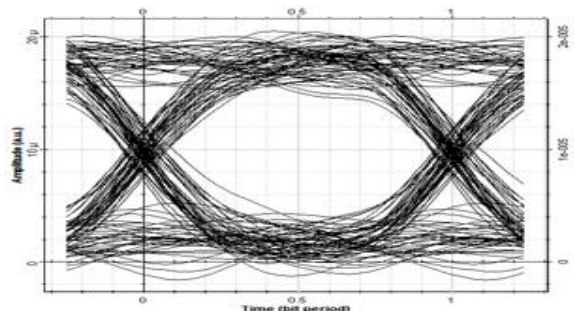

(a) Mode (LG 1 1)

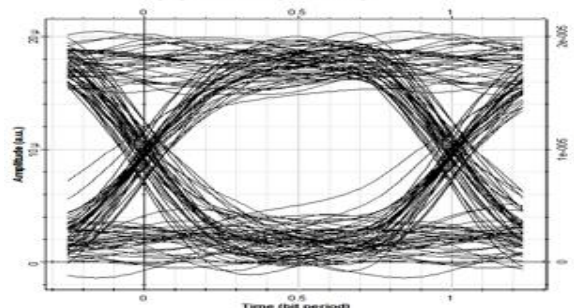

(c) Mode (LG 13 )

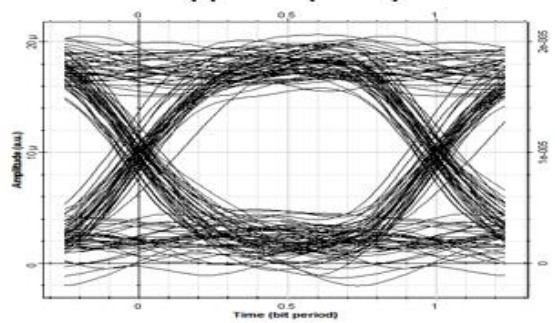

(e) Mode (LG 2 1)

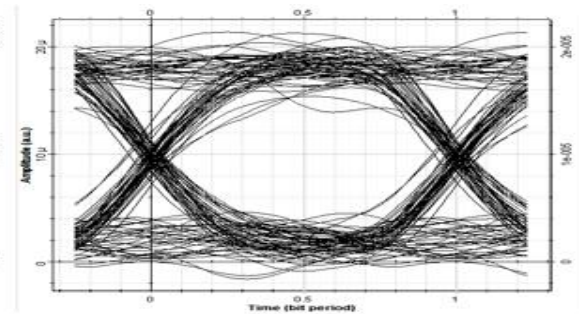

(b) Mode (LG 1 2)

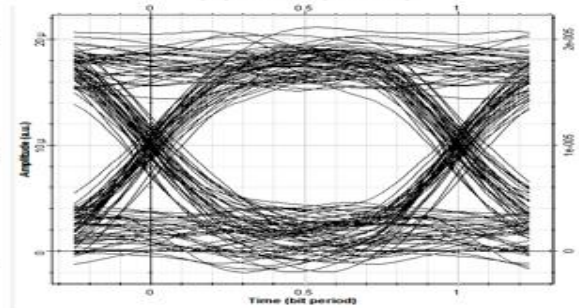

(d) Mode (LG 2 o)

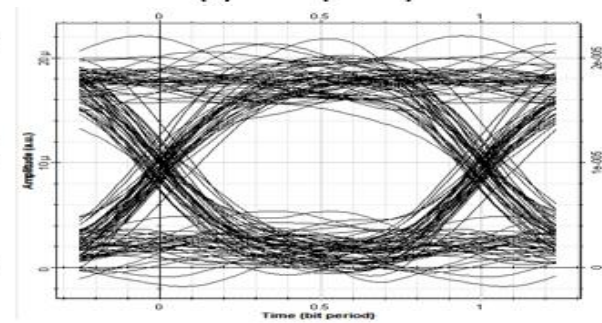

(f) Mode (LG 2 2)

Figure 3. Representing the eye diagrams of last six channels

Table 1. BER for different weather condition with different FSO range

\begin{tabular}{ccccccc}
\hline No & $\begin{array}{c}\text { Weather } \\
\text { Condition }\end{array}$ & $\begin{array}{c}\text { Laser } \\
\text { Power } \\
(\mathrm{dBm})\end{array}$ & $\begin{array}{c}\text { Attenuation } \\
(\mathrm{dB} / \mathrm{km})\end{array}$ & $\begin{array}{c}\text { Max-Link- } \\
\text { Range (Meter) }\end{array}$ & BER & Q-factors \\
\hline 1 & very clear & 1 & 0.15 & $3200 \mathrm{~m}$ & $6.25 \times 10-11$ & 6.432 \\
2 & clear sky & 1 & 0.299 & $3000 \mathrm{~m}$ & $2.13161 \mathrm{e}-11$ & 6.594 \\
3 & light haze & 1 & 0.61 & $2800 \mathrm{~m}$ & $2.31781 \mathrm{e}-10$ & 6.231 \\
4 & heavy haze & 1 & 2.62 & $1900 \mathrm{~m}$ & $1.03215 \mathrm{e}-9$ & 5.992 \\
5 & light rain & 1 & 6.80 & $1200 \mathrm{~m}$ & $4.81945 \mathrm{e}-11$ & 6.472 \\
6 & heavy rain & 1 & 19.77 & $650 \mathrm{~m}$ & $1.18422 \mathrm{e}-9$ & 5.970 \\
\hline
\end{tabular}

Figure 4 represents the curves of the BER. It is seen that for BER 10-11, the transmission distance is up to 650 meters only when it is heavily raining, and improves to 1200 meters when the rain is light. In heavy hazes, it is 1900 meters and improves to 2800 meters for light haze. When the weather is clean, it goes up to 3000 meters. It then improves to 3200 meters in very clear weather condition. These details are shown in Table I. It can be seen that the increase in the distance of FSO transmission decreases the BER curves. Hence, it could be concluded that there is a coefficient in the BER and distance of data transmission. 


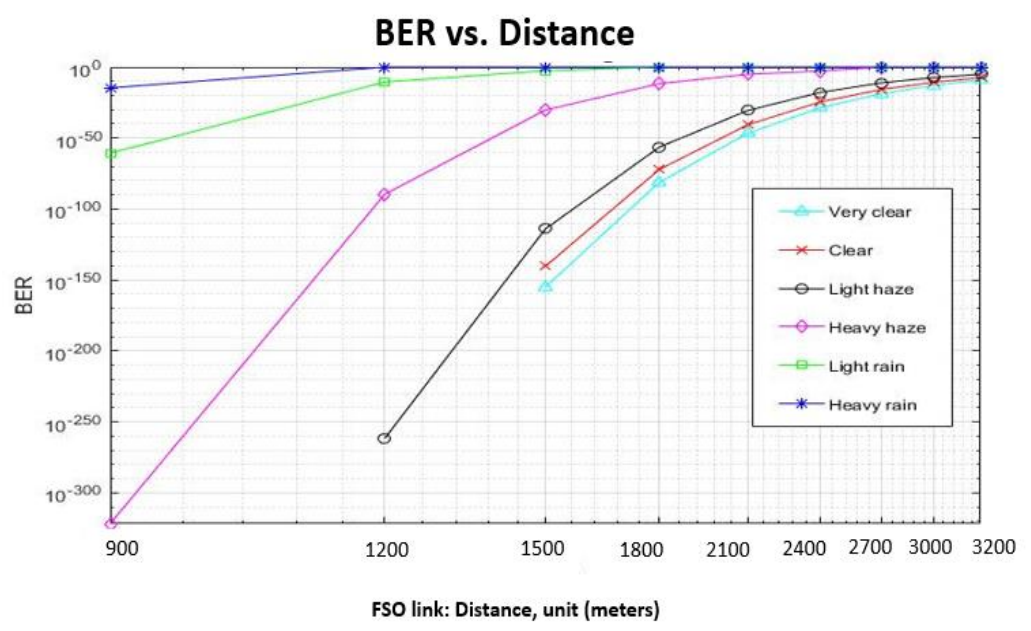

Figure 4. BER result over different range

\section{CONCLUSION}

This paper investigates the performance of BER over different distances of over hybrid FSO in different weather conditions and fiber to the home. The results demonstrate that $10 \times 10 \mathrm{Gbit} / \mathrm{s}$ MDM-FSO system in different weather conditions is effective in improving the quality of data in optical wireless communication system and FTTH. This study has achieved $100 \mathrm{Gbit} / \mathrm{s}$ data transmission over 3200 meters FSO.

\section{REFERENCES}

[1] S. A. Zabidi, W. Al Khateeb, M. R. Islam, and A. W. Naji, "The effect of weather on free space optics communication (FSO) under tropical weather conditions and a proposed setup for measurement," in Computer and Communication Engineering (ICCCE), 2010 International Conference on, 2010, pp. 1-5.

[2] Z. Wang, W.-D. Zhong, S. Fu, and C. Lin, "Performance comparison of different modulation formats over freespace optical (FSO) turbulence links with space diversity reception technique," IEEE Photonics Journal, vol. 1, pp. 277-285, 2009.

[3] D. K. Borah and D. G. Voelz, "Pointing error effects on free-space optical communication links in the presence of atmospheric turbulence," Journal of Lightwave Technology, vol. 27, pp. 3965-3973, 2009.

[4] G. Li, N. Bai, N. Zhao, and C. Xia, "Space-division multiplexing: the next frontier in optical communication," Advances in Optics and Photonics, vol. 6, pp. 413-487, 2014.

[5] M. Y. Aldouri, S. Aljunid, and H. A. Fadhil, "Study of the OCDMA Transmission Characteristics in FSO-FTTH at Various Distances, Outdoor," J. Opt. Commun., vol. 34, pp. 127-133, 2013.

[6] N. Ahmed, S. Aljunid, A. Fadil, R. Ahmad, and M. Rashid, "Hybrid OCDMA/WDM system using complementary detection technique for FTTH access networks," in Industrial Electronics and Applications (ISIEA), 2011 IEEE Symposium on, 2011, pp. 227-230.

[7] C. Fazlina, C. Rashidi, A. Rahman, and S. Aljunid, "Performance Evaluation of a Novel Optimization Sequential Algorithm (SeQ) Code for FTTH Network," in MATEC Web of Conferences, 2017, p. 01002.

[8] M. Islam, N. Ahmed, S. Ali, J. M. Nordin, and A. Sahayadhas, "Detection performance of OCDMA over WDM system for all optical access network," in Communication and Signal Processing (ICCSP), 2016 International Conference on, 2016, pp. 0215-0219.

[9] D. Wang, W. Zhou, Z. Li, Z. Chen, H. Yin, S. Zhu, et al., "Research on free-space optical communication based on time-division multiplexing," in SPIE/COS Photonics Asia, 2016, pp. 100201F-100201F-9.

[10] A. Shahidinejad, I. S. Amiri, and T. Anwar, "Enhancement of indoor wavelength division multiplexing-based optical wireless communication using microring resonator," Reviews in Theoretical Science, vol. 2, pp. 201-210, 2014.

[11] A. Amphawan, S. Chaudhary, and V. Chan, "2 x 20 Gbps-40 GHz OFDM Ro-FSO transmission with mode division multiplexing," Journal of the European Optical Society-Rapid publications, vol. 9, 2014.

[12] A. Amphawan, S. Chaudhary, R. Din, and M. N. Omar, "5Gbps HG 0, 1 and HG 0, 3 optical mode division multiplexing for RoFSO," in Signal Processing \& Its Applications (CSPA), 2015 IEEE 11th International Colloquium on, 2015, pp. 145-149.

[13] S. O. Arik, J. M. Kahn, and K.-P. Ho, "MIMO signal processing for mode-division multiplexing: An overview of channel models and signal processing architectures," IEEE Signal Processing Magazine, vol. 31, pp. 25-34, 2014. 
[14] A. Amphawan, V. Mishra, K. Nisar, and B. Nedniyom, "Real-time holographic backlighting positioning sensor for enhanced power coupling efficiency into selective launches in multimode fiber," Journal of Modern Optics, vol. 59, pp. 1745-1752, 2012.

[15] C. P. Tsekrekos and D. Syvridis, "All-Fiber Broadband $\$\{\backslash \mathrm{rm} \mathrm{LP}\}-\{02\} \$$ Mode Converter for Future Wavelength and Mode Division Multiplexing Systems," IEEE Photonics Technology Letters, vol. 24, pp. 1638$1641,2012$.

[16] neutral Hilbert-space analyzers," vol. 7, p. 44995, 03/27/online 2017.

[17] A. Amphawan and D. O'Brien, "Modal Decomposition of Output Field from Holographic Mode Field Generation in a Multimode Fiber Channel," in IEEE Int. Conf. Photon. (ICP), Langkawi, 2010, pp. 1-5.

[18] A. Amphawan and S. Chaudhary, "Free-space optical mode division multiplexing for switching between millimeter-wave picocells," 2015, pp. 95242H-95242H-6.

[19] Y. Fazea and A. Amphawan, "40Gbit/s MDM-WDM Laguerre-Gaussian Mode with Equalization for Multimode Fiber in Access Networks," Journal of Optical Communications.

[20] A. Amphawan, A. Ghazi, and A. Al-dawoodi, "Free-space optics mode-wavelength division multiplexing system using LG modes based on decision feedback equalization," in EPJ Web of Conferences, 2017, p. 01009.

[21] T. Masunda, A. Amphawan, and A. Al-dawoodi, "Effect of decision feedback equalizer taps on $3 \times 6$-channel mode-wavelength division multiplexing system performance in multimode fiber," in EPJ Web of Conferences, 2017, p. 01012.

[22] A. G. M. Al-Dawoodi, "Neural network equalization scheme to improve channel impulse response at the receiver for optical mode division multiplexing," Universiti Utara Malaysia, 2016.

[23] A. G. M. Al-Dawoodi, "An improved Bees algorithm local search mechanism for numerical dataset," Universiti Utara Malaysia, 2015.

[24] A. G. M. Al-dawoodi and M. Mahmuddin, "An Empirical Study of Double-Bridge Search Move on Subset Feature Selection Search of Bees Algorithm," Journal of Telecommunication, Electronic and Computer Engineering (JTEC), vol. 9, pp. 11-15, 2017.

[25] M. Mahmuddin and A. G. M. Al-dawoodi, "Experimental Study of Variation Local Search Mechanism for Bee Algorithm Feature Selection," Journal of Telecommunication, Electronic and Computer Engineering (JTEC), vol. 9, pp. 103-107, 2017.

[26] O. U. Guide, "RSOFT Design Group," ed: Inc, 2010. 